Proyecciones Journal of Mathematics

Vol. 28, No 2, pp. 169-180, August 2009.

Universidad Católica del Norte

Antofagasta - Chile

\title{
INCLUSION RELATIONS FOR K-UNIFORMLY STARLIKE FUNCTIONS AND SOME LINEAR OPERATOR
}

\author{
HARI SINGH PARIHAR \\ POORNIMA INSTITUTE OF ENG. AND TECH., INDIA \\ Received : February 2009. Accepted : June 2009
}

\begin{abstract}
In this paper, we have established the inclusion relations for $k$ uniformly starlike functions under the $E_{q}^{s}\left(\alpha_{1}\right) f(z)$ operator. These results are also extended to $k$ - uniformly convex functions, close to convex and quasi-convex functions.
\end{abstract}

Subjclass [2000]: Primary 26A33, Secondary 30C45.

Keywords : Starlike, Convex, Linear Operators. 


\section{Introduction}

Let $A$ denote the class of functions that are analytic in the open unit disk $U=\{z \in C:|z|<1\}$

$$
f(z)=z+\sum_{n=2}^{\infty} a_{n} z^{n}, \quad(n \in N) .
$$

A function $f \in A$ is said to be in $\operatorname{UST}(k, \gamma)$, the class of k-uniformly starlike functions of order $\gamma, 0 \leq \gamma<1$, if $f$ satisfies the condition

$$
\Re\left(\frac{z f^{\prime}(z)}{f(z)}\right)>k\left|\frac{z f^{\prime}(z)}{f(z)}-1\right|+\gamma, \quad(k \geq 0) .
$$

A function $f \in A$ is said to be in $\operatorname{UCV}(k, \gamma)$, the class of k-uniformly convex functions of order $\gamma$, if $f$ satisfies the condition

$$
\Re\left\{1+\frac{z f^{\prime \prime}(z)}{f^{\prime}(z)}\right\}>k\left|\frac{z f^{\prime \prime}(z)}{f^{\prime}(z)}\right|+\gamma, \quad(k \geq 0) .
$$

Uniformly starlike and convex functions were first introduced by Goodman[7] and then studied by various authors. For a wealth of references, see RØnning [15].

Setting

$$
\Omega_{k, \gamma}=\left\{u+\iota v ; u>k \sqrt{(u-1)^{2}+v^{2}}+\gamma\right\},
$$

with $p(z)=\frac{z f^{\prime}(z)}{f(z)}$ or $1+\frac{z f^{\prime \prime}(z)}{f^{\prime}(z)}$ and the considering the functions which map $U$ onto the conic domain $\Omega_{k, \gamma}$, such that $1 \in \Omega_{k, \gamma}$, we may rewrite the conditions (1.2) or (1.3) in the form

$$
p(z) \prec q_{k, \gamma}(z) .
$$

We note that the explicit forms of function $q_{k, \gamma}$ for $k=0$ and $k=1$ are

$$
q_{0, \gamma}(z)=\frac{1+(1-2 \gamma) z}{1-z} \text {, and } q_{1, \gamma}(z)=1+\frac{2(1-\gamma)}{\pi^{2}}\left(\log \frac{1+\sqrt{z}}{1-\sqrt{z}}\right)^{2}
$$

For $0<k<1$, we obtain

$$
q_{k, \gamma}(z)=\frac{1-\gamma}{1-k^{2}} \cos \left\{\frac{2}{\pi}(\arccos k) \iota \log \frac{1+\sqrt{z}}{1-\sqrt{z}}\right\}-\frac{k^{2}-\gamma}{1-k^{2}},
$$

and if $k>1$, then $q_{k, \gamma}$ has the form 


$$
q_{k, \gamma}(z)=\frac{1-\gamma}{k^{2}-1} \sin \left\{\frac{\pi}{2 K(k)} \int_{0}^{\frac{u(z)}{\sqrt{k}}} \frac{d t}{\sqrt{1-t^{2} \sqrt{1-k^{2} t^{2}}}}\right\}+\frac{k^{2}-\gamma}{k^{2}-1}
$$

where $u(z)=\frac{z-\sqrt{k}}{1-\sqrt{k} z}$ and $\mathrm{K}$ is such that $k=\cosh \frac{\pi K^{\prime}(z)}{4 K(z)}$.

By virtue of (1.5) and the properties of the domains $\Omega_{k, \gamma}$ we have

$$
\Re(p(z))>\Re\left(q_{k, \gamma}(z)\right)>\frac{k+\gamma}{k+1} .
$$

We define $U C C(k, \gamma, \beta)$ to be the family of functions $f \in A$ such that

$$
\Re\left(\frac{z f^{\prime}(z)}{g(z)}\right)>k\left|\frac{z f^{\prime}(z)}{g(z)}-1\right|+\gamma, \quad(k \geq 0, \quad 0 \leq \gamma<1),
$$

for some $g \in U S T(k, \beta)$.

Similarly, we define $\operatorname{UQC}(k, \gamma, \beta)$ to be the family of functions $f \in A$ such that

$$
\Re\left(\frac{\left(z f^{\prime}(z)\right)^{\prime}}{g^{\prime}(z)}\right)>k\left|\frac{\left(z f^{\prime}(z)\right)^{\prime}}{g^{\prime}(z)}-1\right|+\gamma, \quad(k \geq 0, \quad 0 \leq \gamma<1),
$$

for some $g \in U C V(k, \beta)$.

We note that $\operatorname{UCC}(0, \gamma, \beta)$ is the class of close to convex functions of order $\gamma$ and type $\beta$ and $U Q C(0, \gamma, \beta)$ is the class of quasi convex functions of order $\gamma$ and type $\beta$.

The main object of this paper is to study the inclusion properties of the above mentioned classes under the following linear operator which is defined by Dziok [4].

The Fox-Wright psi function is defined by [5,p.50]

$$
\begin{gathered}
\cdot_{q} \psi_{s}^{*}\left[\begin{array}{ll}
\left(\alpha_{i}, A_{i}\right)_{1, q} \\
\left(\beta_{i}, B_{i}\right)_{1, s} & z
\end{array}\right]=\cdot_{q} \psi_{s}\left[\begin{array}{c}
\left(\alpha_{1}, A_{1}\right), \ldots,\left(\alpha_{q}, A_{q}\right) ; \\
\left(\beta_{1}, B_{1}\right), \ldots,\left(\beta_{s}, B_{s}\right) ;
\end{array}\right] \\
\quad=\sum_{n=0}^{\infty}\left(\prod_{i=1}^{q} \Gamma\left(\alpha_{i}+A_{i} n\right)\right)\left(\prod_{i=1}^{s} \Gamma\left(\beta_{i}+B_{i} n\right)\right)^{-1} \frac{z^{n}}{n !}
\end{gathered}
$$

where $\alpha_{i} \in C(i=1, \ldots, q), \beta_{i} \in C(i=1, \ldots, s)$ and the coefficients $A_{i} \in$ $R_{+}(i=1, \ldots, q)$ and $B_{i} \in R_{+}(i=1, \ldots, s)$ such that 


$$
1+\sum_{i=1}^{s} B_{i}-\sum_{i=1}^{q} A_{i} \geq 0, \quad\left(q, s \in N_{0}=N \cup\{0\}\right)
$$

The normalized Fox-Wright psi function ${ }_{{ }_{q}} \psi_{s}^{*}(z)$ in series form is represented as

$$
\cdot_{q} \psi_{s}^{*}\left[\begin{array}{ll}
\left(\alpha_{i}, A_{i}\right)_{1, q} \\
\left(\beta_{i}, B_{i}\right)_{1, s}
\end{array}\right]=\frac{\Gamma \beta_{1} \ldots \Gamma \beta_{s}}{\Gamma \alpha_{1} \ldots \Gamma \alpha_{q}} \cdot{ }_{q} \psi_{s}\left[\begin{array}{ll}
\left(\alpha_{1}, A_{1}\right), \ldots,\left(\alpha_{q}, A_{q}\right) ; & \\
\left(\beta_{1}, B_{1}\right), \ldots,\left(\beta_{s}, B_{s}\right) ; & z
\end{array}\right]
$$

The ${ }_{q} \psi_{s}(z)$ is a special case of Fox's H-function $H_{k, l}^{m, n}(z)$ (see e.g.[5,p.50]) and ${ }_{q} \psi_{s}^{*}(z)$ is a generalization of the familiar generalized hypergeometric function $\cdot{ }_{q} F_{s}(z)$.

$$
\begin{gathered}
{ }_{q} F_{s}\left[\begin{array}{cc}
\left(\alpha_{i}\right)_{1, q} & \\
\left(\beta_{i}\right)_{1, s} & z
\end{array}\right]={ }_{q} F_{s}\left[\begin{array}{c}
\left(\alpha_{1}\right), \ldots,\left(\alpha_{q}\right) ; \\
\left(\beta_{1}\right), \ldots,\left(\beta_{s}\right) ; z
\end{array}\right] \\
=\sum_{n=0}^{\infty} \frac{\left(\alpha_{1}\right)_{n}, \ldots,\left(\alpha_{q}\right)_{n}}{\left(\beta_{1}\right)_{n}, \ldots,\left(\beta_{s}\right)_{n}} \frac{z^{n}}{n !},
\end{gathered}
$$

where $(\alpha)_{n}$ is the Pochhammer symbol, defined in terms of the gamma function by

$$
(\alpha)_{n}=\frac{\Gamma(\alpha+n)}{\Gamma(\alpha)}
$$

Corresponding to a function $H_{q, s}\left(\alpha_{1}, \ldots, \alpha_{q} ; A_{1}, \ldots, A_{q} ; \beta_{1}, \ldots, \beta_{s} ; B_{1}, \ldots, B_{s} ; z\right)$ is defined by

$$
H_{q, s}\left(\alpha_{1}, \ldots, \alpha_{q} ; A_{1}, \ldots, A_{q} ; \beta_{1}, \ldots, \beta_{s} ; B_{1}, \ldots, B_{s} ; z\right)=z_{\cdot q} \psi_{s}^{*}(z)
$$

We consider a linear operator

$$
\mathrm{E}_{q}^{s}\left(\alpha_{1}, \ldots, \alpha_{q} ; A_{1}, \ldots, A_{q} ; \beta_{1}, \ldots, \beta_{s} ; B_{1}, \ldots, B_{s}\right)
$$

defined by the convolution

$$
\mathrm{E}_{q}^{s}\left(\alpha_{1}, \ldots, \alpha_{q} ; A_{1}, \ldots, A_{q} ; \beta_{1}, \ldots, \beta_{s} ; B_{1}, \ldots, B_{s}\right) f(z)
$$




$$
=H_{q, s}\left(\alpha_{1}, \ldots, \alpha_{q} ; A_{1}, \ldots, A_{q} ; \beta_{1}, \ldots, \beta_{s} ; B_{1}, \ldots, B_{s} ; z\right) * f(z)
$$

For convenience, we write

$$
\mathrm{E}_{q}^{s}\left(\alpha_{i}\right)=\mathrm{E}_{q}^{s}\left(\alpha_{1}, \ldots, \alpha_{q} ; A_{1}, \ldots, A_{q} ; \beta_{1}, \ldots, \beta_{s} ; B_{1}, \ldots, B_{s}\right) \quad(i=1, \ldots, q)
$$

Thus, after some calculations, we get

$$
z\left(A_{i} \mathrm{E}_{q}^{s}\left(\alpha_{i}\right) f(z)\right)^{\prime}=\alpha_{i} \mathrm{E}_{q}^{s}\left(\alpha_{i}+1\right) f(z)-\left(\alpha_{i}-A_{i}\right) \mathrm{E}_{q}^{s}\left(\alpha_{i}\right) f(z) \quad(i=1, \ldots, q)
$$

It should be noted that the linear operator $\mathrm{E}_{q}^{s}\left(\alpha_{i}\right) \quad(i=1, \ldots, q)$ is a generalization of many operators considered earlier. For a special case of this operator Carlson and Shaffer studied this operator under certain restrictions on parameters[2]. A more general operator was studied by Ponnusamy and $\mathrm{R} \emptyset$ nning [24].Also note that special cases of this operator include the Hohlov operator[8], the Ruscheweyh derivative operator [16], the generalized Bernardi-Libera-Livingston linear operator (c.f.[1]) and the Srivastava -Owa fractional derivative operator (c. f. [13], [14]). Many subclasses of analytic functions associated with the operator $\mathrm{E}_{q}^{s}\left(\alpha_{i}\right) \quad(i=$ $1, \ldots, q)$ and its many particular cases were investigated recently by Dziok and Srivastava [3,22,23], Liu and Srivastava [10,11] Gangadharan [18], Liu [9] and others (see also $[14,19,20,21,25]$ ).

We shall need the following Lemmas in the sequel to prove our theorems:

This lemma is given by Eenigenburg,Miller, Mocanu and Reade [6].

Lemma 1.1. Let $\beta, \gamma$ be the complex constants and h be univalently in the unit disk $U$ with $h(0)=c$ and $\Re(\beta h(z)+\gamma)>0$. Let $g(z)=c+\sum_{n=1}^{\infty} p_{n} z^{n}$ be analytic in $\mathrm{U}$. Then

$$
g(z)+\frac{z g^{\prime}(z)}{\beta g(z)+\gamma} \prec h(z) \Rightarrow g(z) \prec h(z)
$$

This lemma is given by Miller and Mocanu[17].

Lemma 1.2. Let $\mathrm{h}$ be the convex in the unit disk $\mathrm{U}$ and let $E \geq 0$. Suppose $B(z)$ is analytic in $\mathrm{U}$ with $\Re(B(z)) \geq E$. If $\mathrm{g}$ is analytic in $\mathrm{U}$ and $g(0)=h(0)$. Then

$$
E z^{2} g^{\prime \prime}(z)+B(z) z g^{\prime}(z)+g(z) \prec h(z) \Rightarrow g(z) \prec h(z) .
$$




\section{Main Results}

Theorem 2.1. Let $\Re\left(\alpha_{1}\right)>A_{1}\left(\frac{1-\gamma}{k+1}\right)$ and $f \in A$. If $\mathrm{E}_{q}^{s}\left(\alpha_{1}+1\right) f \in$ $U S T(k, \gamma)$ then $\mathrm{E}_{q}^{s}\left(\alpha_{1}\right) f \in U S T(k, \gamma)$.

Proof. Setting

$$
p(z)=\frac{z\left(A_{1} \mathrm{Ł}_{q}^{s}\left(\alpha_{1}\right) f(z)\right)^{\prime}}{\mathrm{Ł}_{q}^{s}\left(\alpha_{1}\right) f(z)}
$$

in (1.9), we can write

$$
\alpha_{1} \frac{\mathrm{Ł}_{q}^{s}\left(\alpha_{1}+1\right) f(z)}{\mathrm{Ł}_{q}^{s}\left(\alpha_{1}\right) f(z)}=\frac{z\left(A_{1} \mathrm{Ł}_{q}^{s}\left(\alpha_{1}\right) f(z)\right)^{\prime}}{\mathrm{Ł}_{q}^{s}\left(\alpha_{1}\right) f(z)}+\left(\alpha_{1}-A_{1}\right)=p(z)+\left(\alpha_{1}-A_{1}\right)
$$

Differentiating (2.1) yields

$$
\frac{z\left(A_{1} \mathrm{E}_{q}^{s}\left(\alpha_{1}+1\right) f(z)\right)^{\prime}}{\mathrm{E}_{q}^{s}\left(\alpha_{1}+1\right) f(z)}=p(z)+\frac{z A_{1} p^{\prime}(z)}{p(z)+\left(\alpha_{1}-A_{1}\right)}
$$

From this and argument given in section 1 we may write

$$
p(z)+\frac{z A_{1} p^{\prime}(z)}{p(z)+\left(\alpha_{1}-A_{1}\right)} \prec q_{k, \gamma}(z)
$$

Therefore the theorem follows by Lemma(1.1) and the condition (1.6) since $q_{k, \gamma}$ is univalent and convex in $\mathrm{U}$ and $\Re\left(q_{k, \gamma}\right)>\left(\frac{k+\gamma}{k+1}\right)$ which proves the required result.

Theorem 2.2. Let $\Re\left(\alpha_{1}\right)>A_{1}\left(\frac{1-\gamma}{k+1}\right)$ and $f \in A$. If $\mathrm{E}_{q}^{s}\left(\alpha_{1}+1\right) f \in$ $U C V(k, \gamma)$ then $\mathrm{E}_{q}^{s}\left(\alpha_{1}\right) f \in U C V(k, \gamma)$.

Proof. By vitue of (1.2), (1.3) and Theorem 2.1, we have

$$
\begin{aligned}
\mathrm{\complement}_{q}^{s}\left(\alpha_{1}+1\right) f \in & U C V(k, \gamma) \Leftrightarrow z\left(\mathrm{\complement}_{q}^{s}\left(\alpha_{1}+1\right) f\right)^{\prime} \in U S T(k, \gamma) \\
\Leftrightarrow & \mathrm{E}_{q}^{s}\left(\alpha_{1}+1\right) z f^{\prime} \in U S T(k, \gamma) \\
& \Rightarrow \mathrm{E}_{q}^{s}\left(\alpha_{1}\right) z f^{\prime} \in U S T(k, \gamma)
\end{aligned}
$$




$$
\Leftrightarrow \mathrm{E}_{q}^{s}\left(\alpha_{1}\right) f \in U C V(k, \gamma)
$$

and the proof is complete.

Theorem 2.3. Let $\Re\left(\alpha_{1}\right)>A_{1}\left(\frac{1-\gamma}{k+1}\right)$ and $f \in A$. If $\mathrm{E}_{q}^{s}\left(\alpha_{1}+1\right) f \in$ $U C C(k, \gamma, \beta)$ then $\mathrm{E}_{q}^{s}\left(\alpha_{1}\right) f \in U C C(k, \gamma, \beta)$.

Proof. Since $\mathrm{E}_{q}^{s}\left(\alpha_{1}+1\right) f \in U C C(k, \gamma, \beta)$, by definition, we can write

$$
\frac{z\left(A_{1} \mathrm{E}_{q}^{s}\left(\alpha_{1}+1\right) f\right)^{\prime}(z)}{k(z)} \prec q_{k, \gamma}(z)
$$

for some $k(z) \in U S T(k, \beta)$. For $g$ such that $\mathrm{E}_{q}^{s}\left(\alpha_{1}+1\right) g(z)=k(z)$, we have

$$
\frac{z\left(A_{1} \mathrm{E}_{q}^{s}\left(\alpha_{1}+1\right) f\right)^{\prime}(z)}{\mathrm{E}_{q}^{s}\left(\alpha_{1}+1\right) g(z)} \prec q_{k, \gamma}(z) .
$$

Let $h(z)=\frac{z\left(A_{1} \mathrm{\complement}_{q}^{s}\left(\alpha_{1}\right) f\right)^{\prime}(z)}{\left(\mathrm{\complement}_{q}^{s}\left(\alpha_{1}\right) g(z)\right)}$ and $H(z)=\frac{z\left(\mathrm{\complement}_{q}^{s}\left(\alpha_{1}\right) g\right)^{\prime}(z)}{\mathrm{\complement}_{q}^{s}\left(\alpha_{1}\right) g(z)}$

we observe that $\mathrm{h}$ and $\mathrm{H}$ are analytic in $\mathrm{U}$ and $h(0)=H(0)=1$. Now by Theorem 2.1, $\mathrm{七}_{q}^{s}\left(\alpha_{1}\right) g \in U S T(k, \beta)$ and so $\Re H(z)>\frac{k+\beta}{k+1}$. Also, note that

$$
z\left(A_{1} \mathrm{E}_{q}^{s}\left(\alpha_{1}\right) f\right)^{\prime}(z)=\left(\mathrm{E}_{q}^{s}\left(\alpha_{1}\right) g(z)\right) h(z) .
$$

Differentiating both sides of (2.4)yields

$$
\frac{z\left(A_{1} \mathrm{E}_{q}^{s}\left(\alpha_{1}\right)\left(z f^{\prime}\right)\right)^{\prime}(z)}{\mathrm{Ł}_{q}^{s}\left(\alpha_{1}\right) g(z)}=\frac{z\left(\mathrm{E}_{q}^{s}\left(\alpha_{1}\right) g\right)^{\prime}(z)}{\mathrm{Ł}_{q}^{s}\left(\alpha_{1}\right) g(z)} h(z)+z h^{\prime}(z)=H(z) h(z)+z h^{\prime}(z) .
$$

Now using identity (1.9), we obtain

$$
\begin{aligned}
& \frac{z\left(A_{1} \mathrm{E}_{q}^{s}\left(\alpha_{1}+1\right) f\right)^{\prime}(z)}{\mathrm{Ł}_{q}^{s}\left(\alpha_{1}+1\right) g(z)}=\frac{A_{1}\left(\mathrm{E}_{q}^{s}\left(\alpha_{1}+1\right)\left(z f^{\prime}\right)(z)\right)}{\mathrm{Ł}_{q}^{s}\left(\alpha_{1}+1\right) g(z)} \\
& =\frac{A_{1}\left[z\left(A_{1} \mathrm{E}_{q}^{s}\left(\alpha_{1}\right)\left(z f^{\prime}\right)\right)^{\prime}(z)+\left(\alpha_{1}-A_{1}\right) \mathrm{\complement}_{q}^{s}\left(\alpha_{1}\right)\left(z f^{\prime}\right)(z)\right]}{z\left(A_{1} \mathrm{E}_{q}^{s}\left(\alpha_{1}\right) g\right)^{\prime}(z)+\left(\alpha_{1}-A_{1}\right) \mathrm{E}_{q}^{s}\left(\alpha_{1}\right) g(z)} . \\
& =\frac{A_{1}\left[H(z) h(z)+z h^{\prime}(z)+\frac{\left(\alpha_{1}-A_{1}\right)}{A_{1}} h(z)\right]}{A_{1} H(z)+\left(\alpha_{1}-A_{1}\right)}=h(z)+\frac{z h^{\prime}(z)}{A_{1} H(z)+\left(\alpha_{1}-A_{1}\right)}
\end{aligned}
$$


From (2.3),(2.4) and (2.5) we conclude that

$$
h(z)+\frac{z h^{\prime}(z)}{A_{1} H(z)+\left(\alpha_{1}-A_{1}\right)} \prec q_{k, \gamma}(z) .
$$

Let $E=0$ and $B(z)=\frac{1}{A_{1} H(z)+\left(\alpha_{1}-A_{1}\right)}$, we obtain

$$
\Re(B(z))=\frac{1}{\left|A_{1} H(z)+\left(\alpha_{1}-A_{1}\right)\right|^{2}} \Re\left(A_{1} H(z)+\left(\alpha_{1}-A_{1}\right)\right)>0 .
$$

The above inequality satisfies the conditions required by Lemma (1.2). Hence $h(z) \prec q_{k, \gamma}(z)$ and so the proof is complete.

Using a similar argument to that in Theorem 2.2 we can prove the following Theorem.

Theorem 2.4. Let $\Re\left(\alpha_{1}\right)>A_{1}\left(\frac{1-\gamma}{k+1}\right)$ and $f \in A$. If $\mathrm{E}_{q}^{s}\left(\alpha_{1}+1\right) f \in$ $U Q C(k, \gamma, \beta)$ then $\mathrm{E}_{q}^{s}\left(\alpha_{1}\right) f \in U Q C(k, \gamma, \beta)$.

Finally, we examine the closure property of the above classes of functions under the generalized Bernardi-Libera-Livingston operator $L_{a}(f)$ which is defined by

$$
L_{a}(f)=\frac{a+1}{z^{a}} \int_{0}^{z} t^{a-1} f(t) d t, \quad(a>-1) .
$$

Theorem 2.5. Let $a>\left(\frac{-(k+\gamma)}{k+1}\right)$. If $\mathrm{E}_{q}^{s}\left(\alpha_{1}\right) f \in U S T(k, \gamma)$ so is $L_{a}\left(\mathrm{E}_{q}^{s}\left(\alpha_{1}\right) f\right)$.

Proof. From definition of $L_{a}(f)$ and the linearity of the operator $\mathrm{E}_{q}^{s}\left(\alpha_{1}\right)$ we have

$$
z\left(\mathrm{E}_{q}^{s}\left(\alpha_{1}\right) L_{a}(f)\right)^{\prime}(z)=(a+1) \mathrm{E}_{q}^{s}\left(\alpha_{1}\right) f(z)-a\left(\mathrm{E}_{q}^{s}\left(\alpha_{1}\right) L_{a}(f)\right)(z) .
$$

Substituting $\frac{z\left(\mathrm{E}_{q}^{s}\left(\alpha_{1}\right) L_{a}(f)\right)^{\prime}(z)}{\left(\mathrm{\complement}_{q}^{s}\left(\alpha_{1}\right) L_{a}(f)\right)(z)}=p(z)$ in $(2.6)$ we may write

$$
p(z)=\frac{(a+1) \mathrm{E}_{q}^{s}\left(\alpha_{1}\right) f(z)}{\left(\mathrm{E}_{q}^{s}\left(\alpha_{1}\right) L_{a}(f)\right)(z)}-a .
$$

Differentiating (2.7) gives

$$
\frac{z\left(\mathrm{E}_{q}^{s}\left(\alpha_{1}\right)(f)\right)^{\prime}(z)}{\left(\mathrm{E}_{q}^{s}\left(\alpha_{1}\right) f\right)(z)}=p(z)+\frac{z p^{\prime}(z)}{p(z)+a} .
$$


Now, the theorem follows by Lemma(1.1), since $\Re\left(q_{k, \gamma}(z)+a\right)>0$. A similar argument leads to

Theorem 2.6. Let $a>\left(\frac{-(k+\gamma)}{k+1}\right)$. If $\mathrm{七}_{q}^{s}\left(\alpha_{1}\right) f \in U C V(k, \gamma)$ so is $L_{a}\left(\mathrm{E}_{q}^{s}\left(\alpha_{1}\right) f\right)$.

Theorem 2.7. Let $a>\left(\frac{-(k+\gamma)}{k+1}\right)$. If $\mathrm{E}_{q}^{s}\left(\alpha_{1}\right) f \in U C C(k, \gamma, \beta)$ so is $L_{a}\left(\mathrm{E}_{q}^{s}\left(\alpha_{1}\right) f\right)$.

Proof. By definition there exists a function $k(z)=\left(\mathrm{E}_{q}^{s}\left(\alpha_{1}\right) g\right)(z) \in U S T(k, \beta)$ such that

$$
\frac{z\left(A_{1} \mathrm{E}_{q}^{s}\left(\alpha_{1}\right) f\right)^{\prime}(z)}{\mathrm{Ł}_{q}^{s}\left(\alpha_{1}\right) g(z)} \prec q_{k, \gamma}(z), \quad(z \in U) .
$$

Now from (2.6) we have

$$
\frac{z\left(A_{1} \mathrm{E}_{q}^{s}\left(\alpha_{1}\right) f\right)^{\prime}(z)}{\mathrm{E}_{q}^{s}\left(\alpha_{1}\right) g(z)}=\frac{z\left(A_{1} \mathrm{E}_{q}^{s}\left(\alpha_{1}\right) L_{a}\left(z f^{\prime}\right)\right)^{\prime}(z)+a A_{1} \mathrm{E}_{q}^{s}\left(\alpha_{1}\right) L_{a}\left(z f^{\prime}\right)(z)}{z\left(\mathrm{E}_{q}^{s}\left(\alpha_{1}\right) L_{a}(g(z))\right)^{\prime}(z)+a\left(\mathrm{E}_{q}^{s}\left(\alpha_{1}\right) L_{a}(g)\right)(z)}
$$

Since $\mathrm{E}_{q}^{s}\left(\alpha_{1}\right) g \in U S T(k, \beta)$, by Theorem 2.5, we have $L_{a}\left(\mathrm{E}_{q}^{s}\left(\alpha_{1}\right) g\right) \in$ $U S T(k, \beta)$.

Let $H(z)=\frac{z\left(\mathrm{E}_{q}^{s}\left(\alpha_{1}\right) L_{a}(g)\right)^{\prime}(z)}{\mathrm{\complement}_{q}^{s}\left(\alpha_{1}\right) L_{a} g(z)}$ we note that $\Re(H(z))>\frac{k+\beta}{k+1}$. Now, let $\mathrm{h}$ be defined by

$$
z\left(A_{1} \mathrm{E}_{q}^{s}\left(\alpha_{1}\right) L_{a}(f)\right)^{\prime}=h(z)\left(\mathrm{E}_{q}^{s}\left(\alpha_{1}\right) L_{a}(g)\right)(z) .
$$

Differentiating both sides of (2.10) yields

$\frac{z\left(A_{1} \mathrm{E}_{q}^{s}\left(\alpha_{1}\right)\left(z L_{a}(f)\right)^{\prime}\right)^{\prime}(z)}{\left(\mathrm{E}_{q}^{s}\left(\alpha_{1}\right) L_{a}(g)\right)(z)}=\frac{z\left(\mathrm{E}_{q}^{s}\left(\alpha_{1}\right) L_{a}(g)\right)^{\prime}(z)}{\left(\mathrm{E}_{q}^{s}\left(\alpha_{1}\right) L_{a}(g)\right)(z)} h(z)+z h^{\prime}(z)=H(z) h(z)+z h^{\prime}(z)$

Therefore from (2.9) and (2.11) we obtain

$$
\frac{z\left(A_{1} \mathrm{E}_{q}^{s}\left(\alpha_{1}\right) f\right)^{\prime}(z)}{\left(\mathrm{\complement}_{q}^{s}\left(\alpha_{1}\right)(g)\right)(z)}=\frac{z h^{\prime}(z)+h(z) H(z)+a h(z)}{H(z)+a}
$$


This conjunction with (2.8) leads to

$$
h(z)+\frac{z h^{\prime}(z)}{H(z)+a} \prec q_{k, \gamma}(z) .
$$

Assuming $E=0$ and $B(z)=\frac{1}{H(z)+a}$, we obtain

$\Re(B(z))>0$, if $a>\frac{-(k+\beta)}{k+1}$. Now, we conclude that the proof since the required conditions of lemma 1.2 are satisfied. A similar argument yields

Theorem 2.8. Let $a>\left(\frac{-(k+\gamma)}{k+1}\right)$. If $\mathrm{E}_{q}^{s}\left(\alpha_{1}\right) f \in U Q C(k, \gamma, \beta)$ so is $L_{a}\left(\mathrm{\leftarrow}_{q}^{s}\left(\alpha_{1}\right) f\right)$.

\section{References}

[1] S. D. BERNARDI, Convex and starlike univalent functions, Trans. Amer. Math. Soc., 135, pp. 429-446, (1969).

[2] B. C. CARLSON AND S. B. SHAFFER, Starlike and prestarlike hypergeometric functions, SIAM J. Math. Anal., 15, pp. 737-745, (1984).

[3] J. DZIOK AND H. M. SRIVASTAVA, Certain subclasses of analytic functions associated with the generalized hypergeometric function, Integral Transform Spec.Funct., 14, pp. 7-18, (2003).

[4] J. DZIOK, R. K. RAINA, AND H. M. SRIVASTAVA, Some classes of analytic functions associated with operators on Hilbert space involving Wright's generalized hypergeometric function, Proc. Jangjeon Math. Soc., 7, pp. 43-55, (2004).

[5] H. M. SRIVASTAVA AND H. L. MANOCHA, A treatise on generating functions, Wiley/Halsted,New York, (1984).

[6] P. EEINGENBURG, S. S. MILLER, P. T. MOCANU AND M. D. READE, General Inequalities , (BirkhauseverlagBasel), 64 ISNM, pp. 339-348, (1983).

[7] A. W. GOODMAN, On uniformly starlike functions, $J$. Math. Anal. Appl., 155, pp. 364-370, (1991). 
[8] Yu. E. HOHLOV, Operators and operations in the class of univalent functions, Izv. Vyss. Ucebn. Zaved. Mat., 10, pp. 83-89, (1978).

[9] J. -L. LIU, Strongly starlike functions associated with the Dziok-Srivastava operator, Tamkang J. Math., 35, pp. 37-42, (2004).

[10] J. - L. LIU AND H. M. SRIVASTAVA, Classes of meromorphically multivalent functions associated with the generalized hypergeometric function, Math. Comput. Modelling, 38, pp. 21-34, (2004).

[11] J. -L. LIU AND H. M. SRIVASTAVA, Certain properties of the Dziok-Srivastava operator, Appl. Math. Comput., 159, pp. 485-493, (2004).

[12] S. S. MILLER AND P. T. MOCANU, Differential subordination and inequalities in the complex plane, J. Differential Equations, 67, pp. 199-211, (1987).

[13] S. OWA, On the distortion theorem I, Kyungpook Math. J., 18, pp. 53-58, (1978).

[14] S. OWA AND H. M. SRIVASTAVA, Univalent and starlike generalized hypergeometric functions, Cand. J. Math., 39, pp. 1057-1077, (1987).

[15] F. RØNNING, A survey on uniformly convex and uniformly starlike functions, Ann. Univ. Mariae Curie-Sklodowska, 47 (13), pp. 123-134, (1993).

[16] St. RUSCHEWEYH, New criteria for univalent functions, Proc. Amer. Math. Soc., 49, pp. 109-115, (1975).

[17] S. S. MILLER AND P. T. MOCANU, Differential subordination and inequalities in the complex plane, J. Differential Equations, 67, pp. 199-211, (1987).

[18] A. GANGADHARAN, T. N. SHANMUGAM AND H. M. SRIVASTAVA, : Generalized hypergeometric functions associated with k-uniformly convex functions, Comput. Math. Appl., 44, pp. 1515-1526, (2002). 
[19] H. M. SRIVASTAVA AND S. OWA, : Some characterization and distortion theorems involving fractional calculus, generalized hypergeometric functions, Hadamard products, linear operators and certain subclasses of analytic functions. Nagoya Math. J., 106, pp. 1-28, (1987).

[20] H. M. SRIVASTAVA AND S. OWA(Eds.), : Univalent functions, Fractional calculus, and their applications, Halsted press(Eills Horwood Limited,Chichester)John Wiley and sons, NewYork, Chichester Brisbane and Toronto, (1989).

[21] H. M. SRIVASTAVA AND S. OWA(Eds.), : Current Topics in Analytic Function Theory, World Scientific Publishing Company, Singapore, New Jersey, London and Hong Kong, (1992).

[22] J. DZIOK AND H. M. SRIVASTAVA, : Classes of analytic functions associated with the generalised hypergeometric function, Appl.Math.Comput., 103, pp. 1-13, (1999).

[23] J. DZIOK AND H. M. SRIVASTAVA, : Some subclasses of analytic functions with fixed argument of coefficients associated with the generalized hypergeometric function, Adv.Stud. Contemp. Math., 5, pp. 115-125, (2002).

[24] S. PONNUSAMY and F. RØNNING, : Duality for Hadamard products applied to certain integral transforms, Comlex Variables:Theory Appl., 32, pp. 263-287, (1997).

[25] Y. C. KIM and H. M. SRIVASTAVA, : Fractional integral and other linear operators associated with the Gaussian hypergeometric function, Complex Variables Theory Appl., 34, pp. 293-312, (1997).

\section{Hari Singh Parihar}

Department of Mathematics

Poornima Institute of Engineering and Technology

ISI - 2 RIICO Institutional Area Sitapura

Jaipur 302022

Jaipur

India

e-mail : harisingh.p@rediffmail.com 